Mon. Not. R. Astron. Soc. 000, $113(2002) \quad$ Printed 3 December $2018 \quad$ (MN LATEX style file v2.2)

\title{
The Influence of Streaming Velocities on the Formation of the First Stars
}

\author{
Anna T. P. Schauer ${ }^{1,2} \star \dagger$, Simon C. O. Glover ${ }^{2}$, Ralf S. Klessen ${ }^{2,3}$, Daniel Ceverino ${ }^{2}$ \\ 1 Department of Astronomy, The University of Texas at Austin, Austin, TX 78712, USA \\ 2 Universität Heidelberg, Zentrum für Astronomie, Institut für Theoretische Astrophysik, Albert-Ueberle-Str. 2, 69120 Heidelberg, Germany \\ 3 Universität Heidelberg, Interdiszipliäres Zentrum für Wissenschaftliches Rechnen, Im Neuenheimer Feld 205, 69120 Heidelberg, Germany
}

3 December 2018

\begin{abstract}
How, when and where the first stars formed are fundamental questions regarding the epoch of Cosmic Dawn. A second order effect in the fluid equations was recently found to make a significant contribution: an offset velocity between gas and dark matter, the so-called streaming velocity. Previous simulations of a limited number of low-mass dark matter haloes suggest that this streaming velocity can delay the formation of the first stars and decrease halo gas fractions and the halo mass function in the low mass regime. However, a systematic exploration of its effects in a large sample of haloes has been lacking until now. In this paper, we present results from a set of cosmological simulations of regions of the Universe with different streaming velocities performed with the moving mesh code AREPO. Our simulations have very high mass resolution, enabling us to accurately resolve minihaloes as small as $10^{5} \mathrm{M}_{\odot}$. We show that in the absence of streaming, the least massive halo that contains cold gas has a mass $M_{\text {halo,min }}=5 \times 10^{5} \mathrm{M}_{\odot}$, but that cooling only becomes efficient in a majority of haloes for halo masses greater than $M_{\text {halo, } 50 \%}=1.6 \times 10^{6} \mathrm{M}_{\odot}$. In regions with non-zero streaming velocities, $M_{\text {halo,min }}$ and $M_{\text {halo, } 50 \%}$ both increase significantly, by around a factor of a few for each one sigma increase in the value of the local streaming velocity. As a result, in regions with streaming velocities $v_{\text {stream }} \geqslant 3 \sigma_{\text {rms }}$, cooling of gas in minihaloes is completely suppressed, implying that the first stars in these regions form within atomic cooling haloes.
\end{abstract}

Key words: early universe - dark ages, reionisation, first stars - stars: Population III.

\section{INTRODUCTION}

The first stars in the Universe formed in dark matter minihaloes at high redshift, with masses of $M_{\text {minihalo }} \approx 10^{5}-$ $-10^{7} \mathrm{M}_{\odot}$. Metal-free gas falling into these minihaloes was heated up by shocks and adiabatic compression. In the absence of cooling, the gravitational collapse of the gas would soon have stopped, with the gas becoming fully pressure supported. Previous studies have shown that in order to be able to avoid this fate, the gas must be able to form enough molecular hydrogen $\left(\mathrm{H}_{2}\right)$ to provide efficient cooling on a timescale short compared to the Hubble time (see e.g. the reviews by Bromm 2013, Glover 2013, and references therein). Simple models suggest that the amount of $\mathrm{H}_{2}$ formed in a minihalo is an increasing function of the minihalo mass, while the amount of $\mathrm{H}_{2}$ required for effi-

* anna.schauer@utexas.edu

$\dagger$ NHFP Hubble Fellow cient cooling decreases with minihalo mass (Tegmark et al. 1997). Consequently, there should be some minimum minihalo mass, $M_{\text {halo,min }}$, marking the division between low-mass minihaloes in which $\mathrm{H}_{2}$ cooling is inefficient and the gas does not form stars, and more massive minihaloes in which $\mathrm{H}_{2}$ cooling is efficient and Population III (Pop III) star formation can proceed.

The value of this minimum halo mass is a crucial quantity for understanding the primordial Universe. In $\Lambda$ CDM models, the comoving number density of dark matter haloes increases rapidly with decreasing halo mass $M_{\text {halo }}$. Therefore, the number density of minihaloes capable of hosting Pop III star formation depends strongly on $M_{\text {halo,min }}$, and consequently so does the Pop III star formation rate density at early epochs. This has several important implications.

Firstly, it implies that the size of the contribution that minihaloes make to cosmic reionisation may depend strongly on $M_{\text {halo,min }}$ (see e.g. Ahn et al. 2012). Pop III star-forming minihaloes are important sources of ionizing photons at 
high redshifts, while minihaloes that do not form Pop III stars play an important role by absorbing ionizing photons (Haiman, Abel \& Madau 2001; Barkana \& Loeb 2002, Ciardi et al. 2006). Secondly, the metal pollution history of the Universe also depends on the minimum halo mass for Pop III star formation. Supernovae can efficiently eject large quantities of metal-enriched gas from low-mass minihaloes (Mac Low \& Ferrara 1999, Wise et al. 2012, Smith et al. 2015, see however Chiaki, Susa \& Hirano 2018), and so a lower $M_{\text {halo,min }}$ implies more widespread early metal enrichment of the intergalactic medium. Finally, the value of $M_{\text {halo,min }}$ also has important implications for possible observational tracers of the Pop III epoch, such as the high redshift $21 \mathrm{~cm}$ background (Shapiro et al. 2006, Yue et al. 2009) or the Pop III supernova rate (Magg et al. 2016).

However, despite the importance of $M_{\text {halo,min }}$, there remains a surprising degree of uncertainty regarding its value. Efforts have been made to determine it using simplified toy models (e.g. Tegmark et al. 1997, Trenti \& Stiavelli 2009 Glover 2013), numerical simulations of individual minihaloes (e.g. Fuller \& Couchman 2000, Machacek, Bryan \& Abel 2001) or numerical simulations of large populations of minihaloes (Yoshida et al. 2003), but the scatter between the values obtained by different studies remains significant, as we examine in more detail in Section 3.1 below.

In addition, most studies of $M_{\text {halo,min }}$ have assumed that the baryons and the dark matter are initially at rest with respect to one another. However, it has recently been realized that in general this will not be the case, owing to the existence of residual velocity fluctuations in the baryonic component dating from the epoch when the baryons and photons were strongly coupled (Tseliakhovich \& Hirata 2010, Tseliakhovich, Barkana \& Hirata 2011). These residual velocity fluctuations result in a systematic motion of the baryons relative to the dark matter prior to the onset of non-linear structure formation. They are coherent on a scale of several comoving $\mathrm{Mpc}(\mathrm{cMpc})$ and have a rootmean-squared value of $\sigma_{\text {rms }} \approx 30 \mathrm{~km} \mathrm{~s}^{-1}$ at $z \approx 1100$.

Although this relative velocity decreases as $v_{\text {stream }} \sim$ $(1+z)$ as the Universe expands, it nevertheless has profound effects on the formation of dark matter minihaloes. Streaming of baryons with respect to the dark matter leads to a reduced baryon fraction in the haloes (Naoz, Yoshida \& Gnedin 2012) and a lower halo number density (Tseliakhovich, Barkana \& Hirata 2011; Naoz, Yoshida \& Gnedin 2013), as it is harder for baryons to settle into the host dark matter haloes. As a consequence, Pop III star formation is delayed (Greif et al. 2011, Fialkov et al. 2012, O'Leary \& McQuinn 2012 Hirano et al. 2018, although see also Stacy, Bromm \& Loeb 2011 for a dissenting view).

Simulations of the impact of streaming on the cooling and collapse of gas in individual minihaloes suggest that it leads to an increase in $M_{\text {halo,min. If true, this will have a }}$ clear impact on the high redshift $21 \mathrm{~cm}$ background (Fialkov \& Barkana 2014, Fialkov 2014) and in extreme regions (i.e. regions with streaming velocities a few times greater than the rms value), it may also help to create the environment required for the formation of direct collapse black holes (Tanaka \& Li 2014, Latif, Niemeyer \& Schleicher 2014 Schauer et al. 2017). However, as yet no numerical studies have quantified the relationship between $v_{\text {stream }}$ and $M_{\text {halo,min }}$ for a large sample of minihaloes over a broad range of redshifts. It is this lack that we attempt to remedy in our current paper.

We present here the results of a set of high resolution cosmological simulations carried out with streaming velocities $v_{\text {stream }}=0,1,2$, and 3 times $\sigma_{\text {rms }}$. Several thousand minihaloes form in each simulation by our final redshift $z=14$, allowing us to examine the impact of the streaming on a large statistical sample of minihaloes. In contrast to previous studies (e.g. Naoz, Yoshida \& Gnedin 2012, 2013. Popa et al. 2016), we also include a primordial chemistry network in our simulations, allowing us to investigate the thermal evolution of the gas. We can therefore not only investigate the gas fraction in our studies, but also quantify how much cold, dense gas is available for Pop III star formation in the haloes. In most of our simulations, we use a very high resolution of $20 \mathrm{M}_{\odot}$ per gas cell, allowing us to follow the collapse of the gas up to densities of $n>10^{2} \mathrm{~cm}^{-3}$ in every minihalo in which cooling and collapse takes place. We are therefore able to derive quantitative and statistically robust results about the influence of streaming velocities on first star formation.

Our paper is structured as follows: we present our set of simulations in Section 2. In Section 3, we analyse the results of our study, starting with the simulation without streaming velocities in Section 3.1. In Section 3.2 we then present the results for simulations with streaming velocities of 1,2 or $3 \sigma_{\text {rms }}$. We give our conclusions in Section 4 .

\section{METHOD}

\section{$2.1 \quad$ Numerical method}

Our cosmological simulations are carried out using the moving-mesh code AREPO (Springel 2010). AREPO solves the equations of hydrodynamics on an unstructured mesh defined by the Voronoi tessellation of a set of mesh-generating points that move with the flow of the gas. Dark matter is included using a Barnes \& Hut (1986) oct-tree.

To model the chemical and thermal evolution of the gas, we use the same primordial chemistry network and cooling function as in Schauer et al. (2017). These are based on the versions implemented in AREPO by Hartwig et al. (2015a), but we have updated them in several respects. Most significantly, we have included a simplified model of deuterium chemistry, focused on the formation and destruction of hydrogen deuteride, HD. In addition, we have updated several of the chemical rate coefficients used within the model. Full details regarding these updates are given in Appendix A

\subsection{Initial conditions}

Our simulations are initialised at $z=200$. Their details are summarised in Table 1. The initial conditions for the dark matter are created with MUSIC (Hahn \& Abel 2011), using the transfer functions of Eisenstein \& Hu (1998). The baryons are assumed to initially trace the dark matter density distribution. We assume a $\Lambda \mathrm{CDM}$ cosmology and use cosmological parameters derived from PLANCK observations of the CMB, namely $h=0.6774, \Omega_{0}=0.3089, \Omega_{\mathrm{b}}=0.04864$, $\Omega_{\Lambda}=0.6911, n=0.96$ and $\sigma_{8}=0.8159$ (Planck Collaboration et al. 2016). 
Streaming velocities at first star formation

When setting up our initial conditions, we neglect differences in the density fluctuations for gas and dark matter. This can lead to increased baryon fractions in minihaloes and to a lower the minimum halo mass (see e.g. $\mathrm{NaOz}$ \& Barkana 2005, Naoz, Barkana \& Mesinger 2009, Naoz, Yoshida \& Barkana 2011). However, for our models the effect is less than <1\% compared to work by Popa et al. 2016 who treat this effect more carefully. To do better, a more sophisticated treatment is necessary, e.g. by using codes such as BCCOMICS (Ahn \& Smith 2018) or creating transfer functions based on a modified version of CMBFAST (Seljak \& Zaldarriaga 1996, Popa et al. 2016) for setting up initial conditions.

In our runs without streaming, the initial velocity field of the baryons is the same as that of the dark matter. In the runs that include streaming, we add a constant velocity offset, arbitrarily chosen to be in the $x$-direction, with a magnitude $v_{\text {stream }}$. We run simulations with $v_{\text {stream }}=6,12$, and $18 \mathrm{~km} \mathrm{~s}^{-1}$, corresponding to values 1,2 and 3 times $\sigma_{\text {rms }}$, the root-mean-squared streaming velocity at $z=200$. In our initial conditions, we ignore the effect of a position shift of the baryons with respect to the dark matter due to the streaming, as Naoz, Yoshida \& Gnedin (2012) have shown that this is unimportant at $z \gg 15$ and is completely erased by the time $z \sim 15$.

The naming convention for our simulations is simple. Each simulation has a name of the form $\mathrm{v} N$, where $N$ denotes the streaming velocity in units of $\sigma_{\text {rms }}$; thus, v0 corresponds to a run with $v_{\text {stream }}=0$. Most of the simulations are carried out with a box size of $(1 \mathrm{cMpc} / h)^{3}$. However, for the case with $v_{\text {stream }}=3 \sigma_{\text {rms }}$, we have carried out an additional simulation with a significantly larger box size, $(4 \mathrm{Mpc} / h)^{3}$, which we name v3_big.

All simulations are performed with $1024^{3}$ dark matter particles and initially have $1024^{3}$ Voronoi mesh cells to represent the gas. This corresponds to a dark matter particle mass of $99 \mathrm{M}_{\odot}$ and an average initial mesh cell mass of $18.6 \mathrm{M}_{\odot}$ in the simulations with a box size of $(1 \mathrm{Mpc} / h)^{3}$. We have verified that this mass resolution is sufficient to obtain numerically converged results, as discussed in more detail in Appendix $B$ The corresponding values in the run with a larger box are larger by a factor of $4^{3}=64$.

We use ths standard AREPO refinement scheme, in which the code refines or de-refines cells as necessary to keep the mass of the gas close to the initial cell mass. At high densities, we prevent run-away collapse by switching off refinement once the cell volume drops below $0.1 h^{-3} \mathrm{cpc}^{3}$. The corresponding maximum density is redshift dependent, but is typically a few times $10^{6} \mathrm{~cm}^{-3}$. Since we are interested in this paper in the behaviour of gas at densities orders of magnitude lower than this value, this choice does not affect our results.

\subsection{Halo selection}

We select haloes via a standard friends-of-friends algorithm. We have verified that our results do not change significantly if we look only at the first subhalo in each halo that is actually gravitationally bound (see Appendix C).

At each output redshift, we examine every halo and identify those containing cold gas. In order to be counted as

\begin{tabular}{lcccc} 
Name & $\begin{array}{c}\text { Box size } \\
{[\mathrm{cMpc} / h]}\end{array}$ & $\begin{array}{c}v_{\text {stream }} \\
{\left[\mathrm{km} \mathrm{s}^{-1}\right]}\end{array}$ & $\begin{array}{c}M_{\text {gas }} \\
{\left[\mathrm{M}_{\odot}\right]}\end{array}$ & $\begin{array}{c}M_{\mathrm{DM}} \\
{\left[\mathrm{M}_{\odot}\right]}\end{array}$ \\
\hline v0 & 1 & 0 & 18.6 & 99 \\
v1 & 1 & 6 & 18.6 & 99 \\
v2 & 1 & 12 & 18.6 & 99 \\
v3 & 1 & 18 & 18.6 & 99 \\
v3_big & 4 & 18 & 1190 & 6360 \\
\hline
\end{tabular}

Table 1. Overview of our simulations. Note that $v_{\text {stream }}$ gives the strength of the streaming velocity at $z=200$. $M_{\text {gas }}$ and $M_{\mathrm{DM}}$ are the initial mesh cell gas mass and dark matter particle mass, respectively.

"cold and dense" for our purposes, gas needs to fulfill the following three criteria:

- Temperature $T \leqslant 500 \mathrm{~K}$,

- Number density $n \geqslant 100 \mathrm{~cm}^{-3}$,

- Fractional $\mathrm{H}_{2}$ abundance $x_{\mathrm{H}_{2}} \geqslant 10^{-4}$.

These criteria allow us to distinguish between gas which has formed $\mathrm{H}_{2}$, cooled and begun to undergo gravitational collapse, and gas which instead has a low temperature simply because it has not yet virialized. Our selection is insensitive to the values chosen for the temperature and molecular hydrogen abundance criteria, provided we choose a temperature lower than the typical halo virial temperature and larger than the minimum temperature reachable by $\mathrm{H}_{2}$ cooling, $T \sim 200 \mathrm{~K}$, and an $\mathrm{H}_{2}$ abundance that is considerably larger than the abundance in the undisturbed intergalactic medium, $x_{\mathrm{H}_{2}} \sim 10^{-6}$ (Hirata \& Padmanabhan 2006). We assume that gas denser than our threshold has reached the "point of no return" beyond which the gas cannot be stopped from collapsing, implying that the halo will form stars shortly afterwards. Any chosen density threshold is to some extent arbitrary. In fact, the minimum halo mass, which we investigate in Section 3 , does change by a few tens of percent for lower or higher number densities. We define the cold mass, $M_{\text {cold }}$, as the sum of the gas masses in all of the mesh cells that fulfill the three criteria above. A halo that contains at least one such gas cell is counted to be "cold" and contributes to the number of cold gas haloes, $N_{\text {cold }}$.

\section{RESULTS}

\subsection{No streaming velocities}

We begin by analysing the evolution of haloes in a patch of the Universe with zero streaming velocity. Figure 11 shows the cumulative halo mass function $N(>M)$ plotted for several different redshifts in the range $14 \leqslant z \leqslant 24$. We also show the cumulative cold halo mass function $N_{\text {cold }}(>M)$ for all haloes that contain at least one cold, dense gas cell. At redshifts $z \leqslant 20$, the most massive haloes are always cold, whereas smaller haloes with masses less than a few times $10^{5} \mathrm{M}_{\odot}$ are never cold, independent of redshift.

Figure 2 shows the cold gas mass $M_{\text {cold }}$ plotted against the halo mass $M_{\text {halo }}$ for all haloes that contain at least one cold gas cell at redshift $z=15$. In most of the haloes, there is a clear correlation between cold gas mass and halo mass 


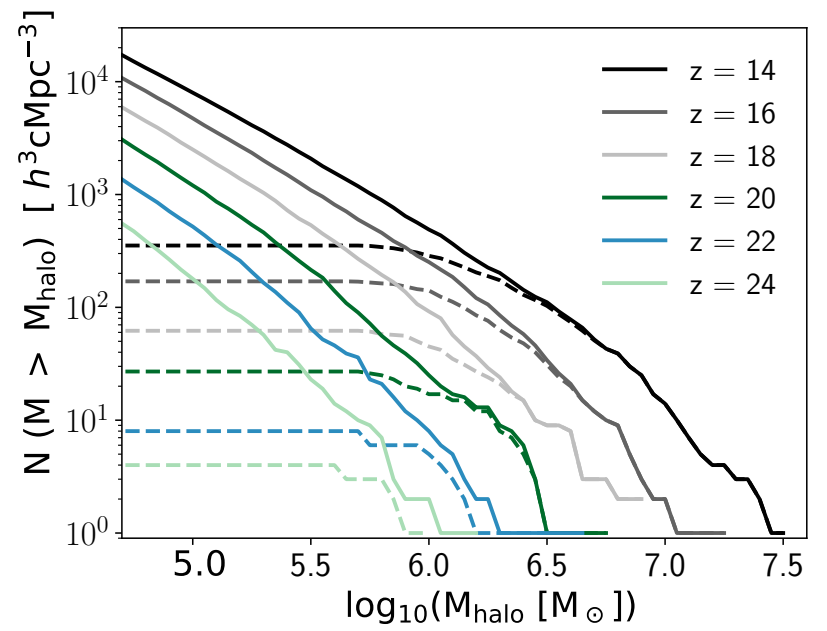

Figure 1. Cumulative halo mass function for all haloes (solid lines) and cold haloes (dashed lines) for redshifts from $z=14$ to $z=24$ for the simulation without streaming.

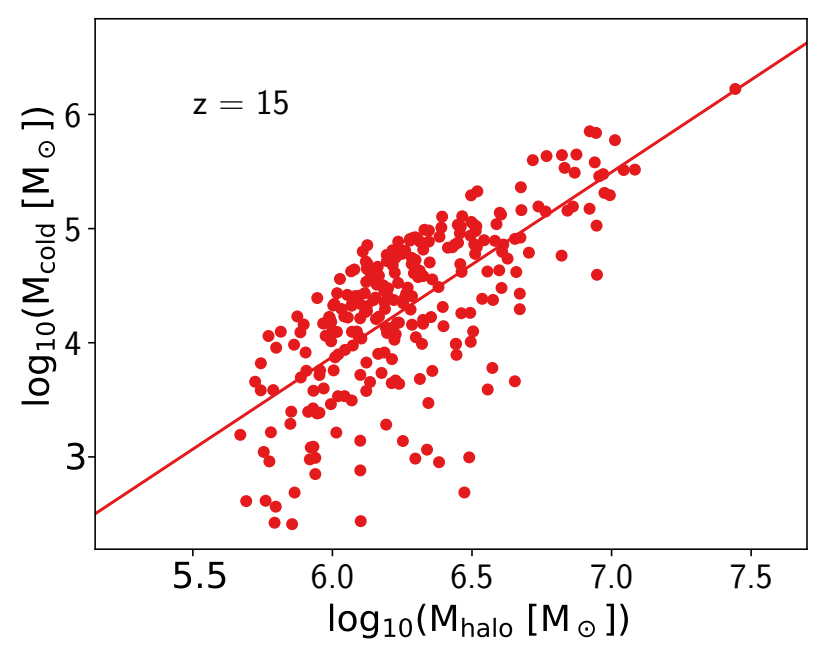

Figure 2. Cold gas mass $M_{\text {cold }}$ plotted as a function of halo mass $M_{\text {halo }}$ for the simulation without streaming velocity at $z=15$. The solid red line provides a fit to the data points.

that can be fit with the function

$$
M_{\text {cold }}=7.55 \times 10^{3} \mathrm{M}_{\odot} \times\left(\frac{M_{\text {halo }}}{10^{6} \mathrm{M}_{\odot}}\right)^{1.6},
$$

indicated by the red line in the plot. The mass in stars is often inferred directly from the halo mass in semi-analytical models of Pop. III star formation (see e.g. Hartwig et al. 2015b. Magg et al. 2018), and we provide the amount of cold gas available as a function of halo mass as an in between step. We have computed similar fits for all of the simulations that we have carried out (see Appendix D). However, it is clear from the Figure that this relationship is at best a crude approximation. In particular, there are a number of haloes in the mass range $10^{5.7}<M<10^{6.5} \mathrm{M}_{\odot}$ that sit far below the fit, with cold gas masses $M_{\text {cold }}<10^{3} \mathrm{M}_{\odot}$.

The relation between the cold gas mass and the halo mass does not vary strongly with redshift over the range of redshifts studied here. Figure 3 shows the cold gas mass-halo

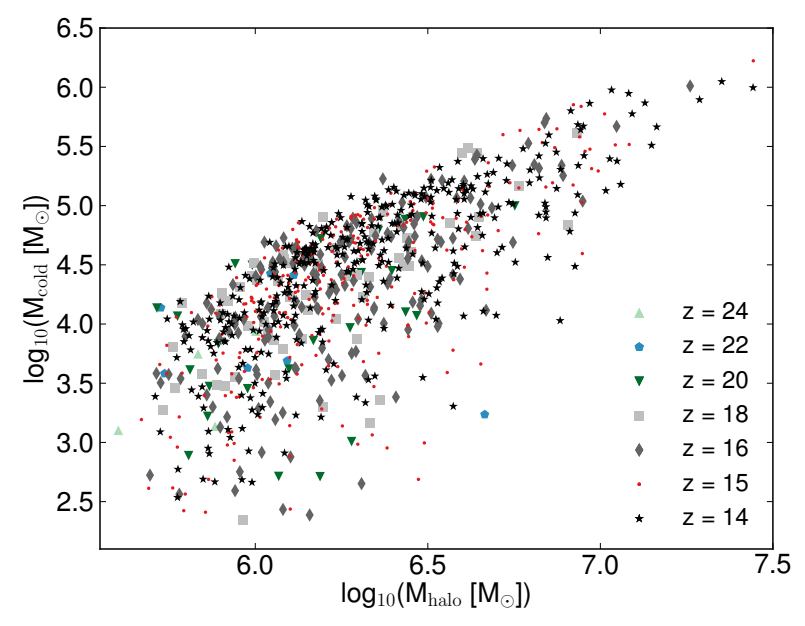

Figure 3. Cold gas mass $M_{\text {cold }}$ plotted as a function of halo mass $M_{\text {halo }}$ for the simulation without streaming velocity at redshifts in the range $14 \leqslant z \leqslant 24$.

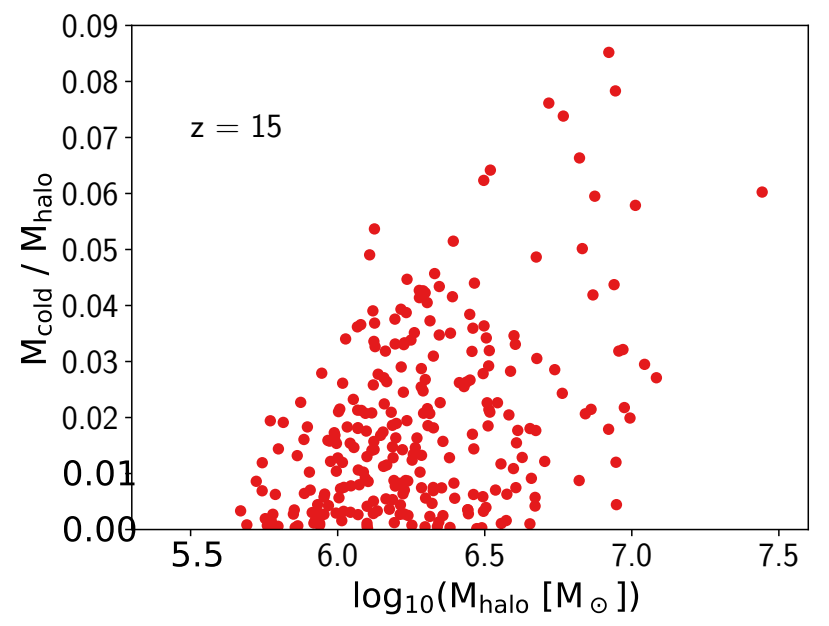

Figure 4. Cold gas fraction $M_{\text {cold }} / M_{\text {halo }}$ plotted as a function of halo mass $M_{\text {halo }}$ for the simulation without streaming velocity at $z=15$. Values are plotted only for haloes that contain at least one cold gas cell.

mass relation for the same simulation for a number of different redshifts in the range $14 \leqslant z \leqslant 24$. At redshifts $z=24$ (light green triangles) and $z=22$ (blue pentagons), we are limited by small number statistics as our simulation includes only 4 and 8 haloes that contain cold gas, respectively. For redshifts $z \leqslant 20$, the cold mass-halo mass relations for different redshifts agree well.

As expected, larger haloes contain more cold gas, but in part this is because they contain more gas in total. We can more easily examine whether cooling is more efficient in these haloes by plotting the cold gas fraction $M_{\text {cold }} / M_{\text {halo }}$ as a function of halo mass $M_{\text {halo }}$, as shown in Figure 4. We see a weak correlation between cold gas fraction and halo mass. Larger haloes tend to have higher cold gas fractions, but the scatter is large.

The cold gas fraction is similar for higher redshifts as can be seen in Figure 5. The main difference arises from the fact that the haloes forming at higher redshifts are less 


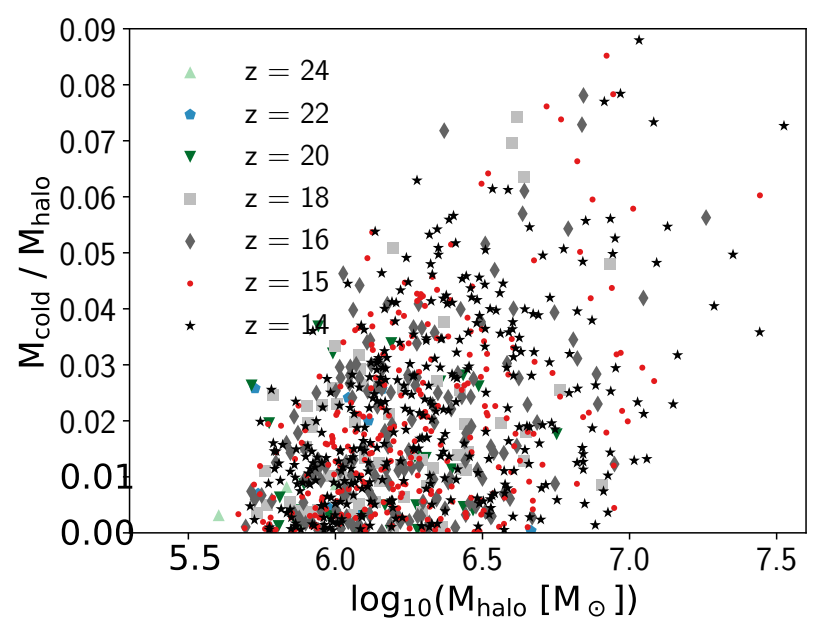

Figure 5. Cold gas fraction $M_{\text {cold }} / M_{\text {halo }}$ plotted as a function of halo mass $M_{\text {halo }}$ for the simulation without streaming velocity for redshifts in the range $14 \leqslant z \leqslant 24$. Values are plotted only for haloes that contain at least one cold gas cell.

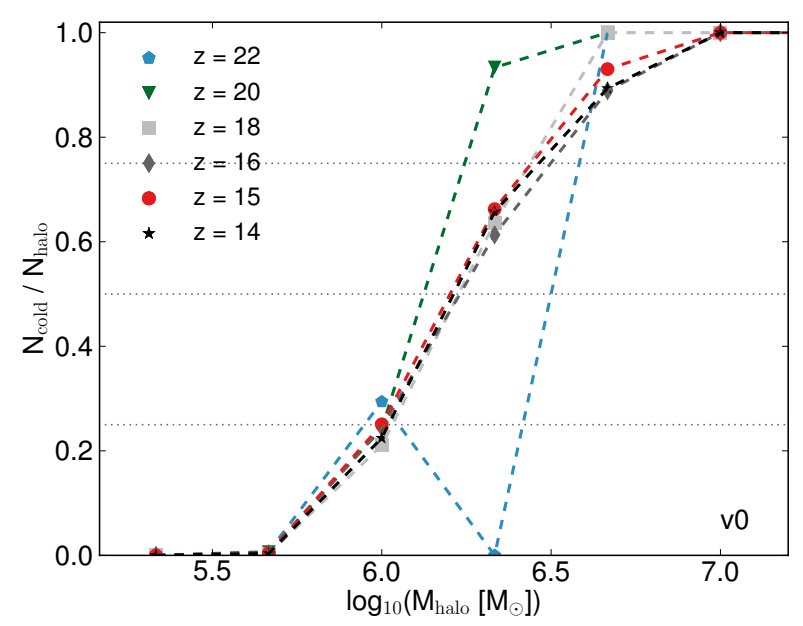

Figure 6. Fraction of haloes of a given mass that contain cold gas plotted as function of halo mass for different redshifts in the range $14 \leqslant z \leqslant 22$. The dotted, grey horizontal lines mark the $25 \%$, $50 \%$ and $75 \%$ transitions of $N_{\text {cold }} / N_{\text {halo }}$ from fewer to more cold haloes. $N_{\text {cold }} / N_{\text {all }}$ generally increases with halo mass. At redshift $z=22$ we are limited by low number statistics, since only 8 haloes in the simulation contain any cold gas at this redshift.

massive and thus the diagram is less populated at higher halo masses for higher redshifts.

Another interesting quantity is the minimum halo mass $M_{\text {halo,min }}$ required for haloes to contain cold gas. Since gas cooling and the consequent loss of thermal pressure support is a necessary condition for star formation in these low-mass haloes, this is also the minimum halo mass required for Pop. III star formation. There appears to be a clear edge to the distribution of points on the left-hand side of Figures $2,3,4$ and 5 that corresponds to a minimum halo mass. To quantify this, we show the fraction of haloes with at least one cold gas particle, $N_{\text {cold }}$, over the number of haloes in the mass bin, $N_{\text {all }}$, for different redshifts in Figure 6. At all times, the fraction $N_{\text {cold }} / N_{\text {all }}$ increases with halo mass $M_{\text {halo }}$. At redshifts

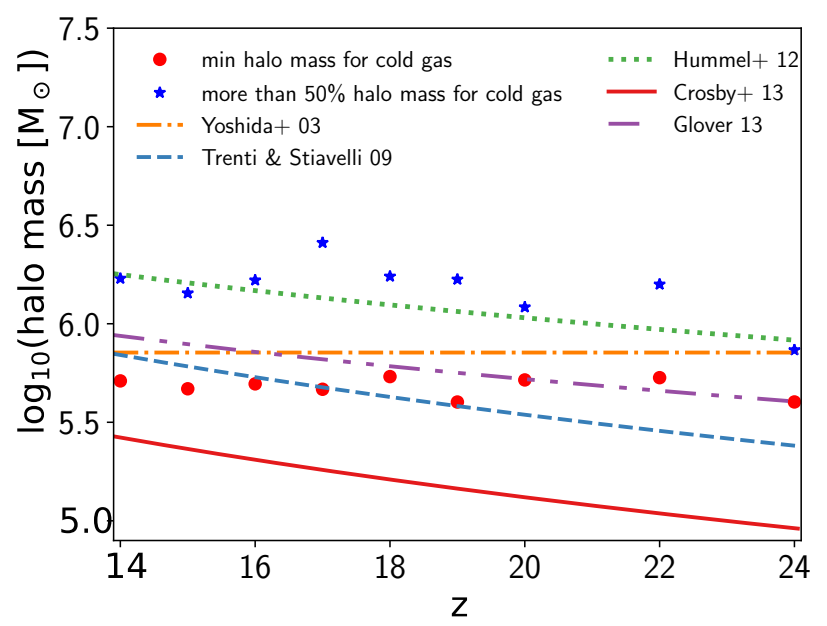

Figure 7. Minimum halo mass $M_{\text {halo,min }}$ for a halo to contain cold gas and halo mass $M_{\text {halo,50\% }}$ above which more than $50 \%$ of all haloes harbour cold, dense gas plotted as a function of redshift. Over-plotted are data from the literature from Yoshida et al. (2003), Trenti \& Stiavelli (2009), Hummel et al. (2012), Crosby et al. (2013), and Glover (2013). Note that the results from Yoshida et al. (2003) are empirical results from a cosmological simulation, while the other values are from simplified models of cooling and collapse in high redshift minihaloes.

$z \leqslant 22$, there is always a minimum halo mass above which more than $50 \%$ of all haloes harbour cold gas. The transition happens at $M_{\text {halo }} \approx 1.6 \times 10^{6} \mathrm{M}_{\odot}$ and is independent of redshift.

We show these two masses, the minimum halo mass $M_{\text {halo,min }}$ and the halo mass above which $50 \%$ or more of haloes contain cold gas $M_{\text {halo,50\% }}$ in Figure 7 In addition, we also show several models from the literature for comparison.

Simulations by Yoshida et al. 2003, orange dot-dashed line) find a minimum halo mass of $\approx 7 \times 10^{5} \mathrm{M}_{\odot}$, which is about $50 \%$ larger than our value. As their simulation was carried out at lower resolution (their best resolution is about a factor of two lower than ours, $m_{\text {gas }} \approx 42 \mathrm{M}_{\odot}$ ), used an earlier and less accurate treatment of the $\mathrm{H}_{2}$ cooling function, and also adopted a higher density threshold for their cold gas $\left(n \geqslant 5 \times 10^{2} \mathrm{~cm}^{-3}\right)$, this small discrepancy is not surprising.

Other authors have used semi-analytic models to predict the minimum halo mass. Some of these models produce values very close to ours at the middle of our range of redshifts (e.g. Trenti \& Stiavelli 2009, Glover 2013), but it is clear that all of the semi-analytical models predict an inverse relationship between $M_{\text {halo,min }}$ and redshift that is not seen in our simulation or in the Yoshida et al. (2003) results.

Finally, it is important to note that $M_{\text {halo,min }}$ appears to be quite sensitive to density threshold used to identify haloes with cold, dense gas, changing by tens of percent for changes in the threshold of a factor of a few. $M_{\text {halo, } 50 \%}$, on the other hand, has little sensitivity to the choice of threshold.

\subsection{Streaming velocities}

We now examine how increasing the streaming velocity affects the ability of gas to cool and form stars within low-mass 


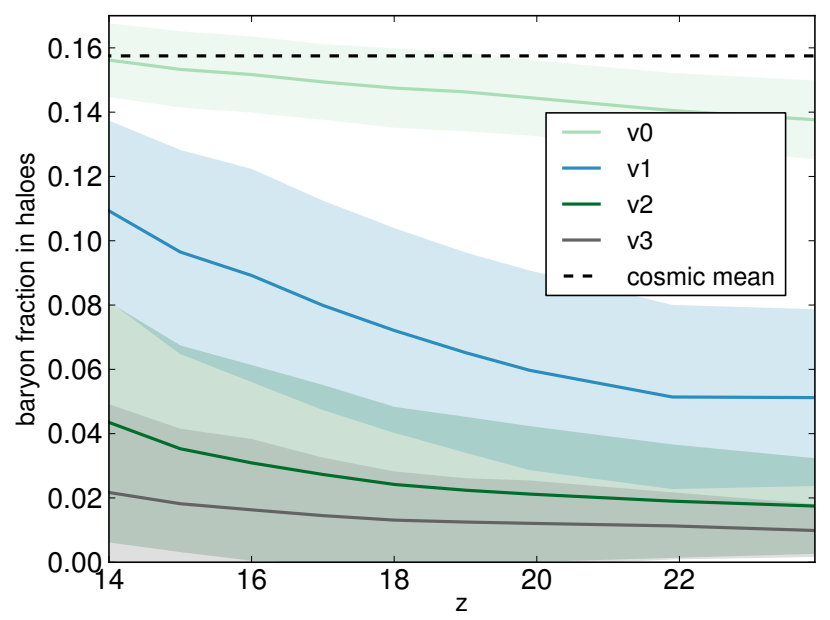

Figure 8. Baryon fraction in haloes with masses of $M_{\text {halo }} \geqslant$ $10^{5} \mathrm{M}_{\odot}$ as a function of redshift. In the case of zero streaming velocity (light green), the value approaches the cosmic mean (dashed line). For all simulations with non-zero streaming velocities (blue, dark green, grey), it is well below that value. The shaded regions show the respective standard deviations.

haloes. We start by looking at two effects that have previously been studied by other authors and that we can confirm with our own simulations.

First, a non-zero streaming velocity makes it harder for baryons to settle into low-mass dark matter haloes. This can be quantified by examining the behaviour of the baryon fraction $M_{\text {bar }} / M_{\text {halo }}$ as a function of redshift, as we do in Figure 8. Here we show the evolution with redshift of the mean and the standard deviation of the baryon fraction in haloes with mass $M>10^{5} \mathrm{M}_{\odot}$ or higher in runs v0, v1, v2 and v3. For the range of redshifts examined here, the Jeans mass $M_{\mathrm{J}} \ll 10^{5} \mathrm{M}_{\odot}$, and so in the absence of streaming we expect the baryon fraction to be close to the cosmic mean of $\Omega_{\mathrm{b}} / \Omega_{0} \approx 0.16$, as is the case in run v0. However, as we increase the streaming velocity, the baryon fraction decreases, reaching values as low as 0.01 at early times in the v3 run. This decrease in gas fraction was predicted by Tseliakhovich \& Hirata (2010) and has been noted in previous simulations by several authors (e.g. Tseliakhovich, Barkana \& Hirata 2011, Naoz, Yoshida \& Gnedin 2013). We confirm the effect with our own high resolution data.

A second large-scale effect follows as a consequence: the halo mass function decreases in simulations of non-zero streaming velocity regions owing to the decreased contribution of baryons to the self-gravity of overdense regions. We show how the influence of streaming velocities lowers the cumulative halo mass function $N\left(>M_{\text {halo }}\right)$ at redshift $z=15$ in Figure 9 for all of the simulations carried out using the $(1 \mathrm{Mpc} / h)^{3}$ simulation volume. The suppression in our simulations is on the order of $10 \%$ to $25 \%$ in the mass range of $M_{\text {halo }}=10^{5}-10^{6} \mathrm{M}_{\odot}$ at $z=15$ for each $\sigma_{\text {rms }}$ increase in the streaming velocity. Naoz, Yoshida \& Gnedin (2012) see a decrease by $15 \%$ and $50 \%$ for $1.7 \sigma_{\mathrm{rms}}$ and $3.4 \sigma_{\mathrm{rms}}$ regions, respectively at redshift $z=19$. Popa et al. (2016) find a similar decrease of small objects with $M_{\text {halo }} \leqslant 10^{5} \mathrm{M}_{\odot}$ of $\sim 60 \%$ at redshift $z=20$. Our results are therefore in good agreement with other recent studies.

We also note that the overall normalisation of our halo

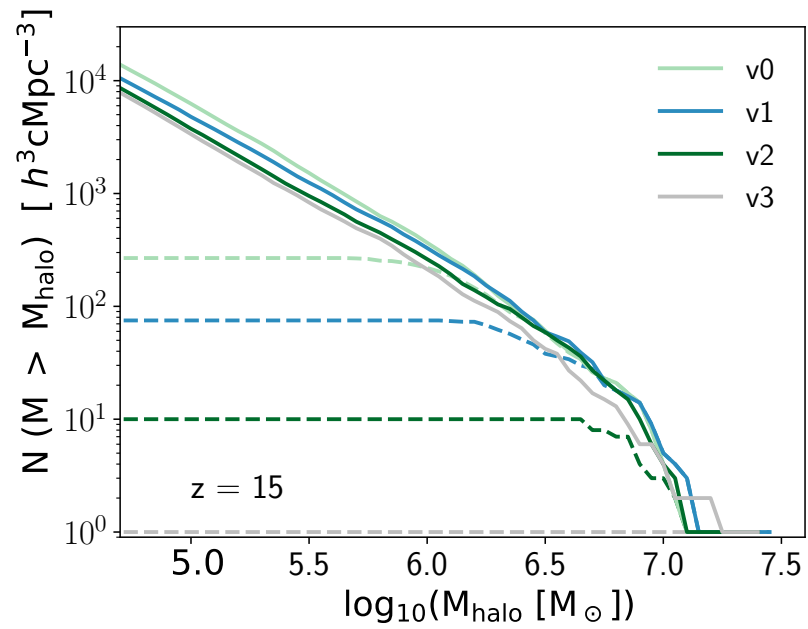

Figure 9. Cumulative halo mass function $N\left(>M_{\text {halo }}\right)$ at redshift $z=15$ for simulations $\mathrm{v} 0, \mathrm{v} 1, \mathrm{v} 2$ and $\mathrm{v} 3$. The total number of haloes is shown as a solid line and the number of haloes with cold gas as a dashed line.

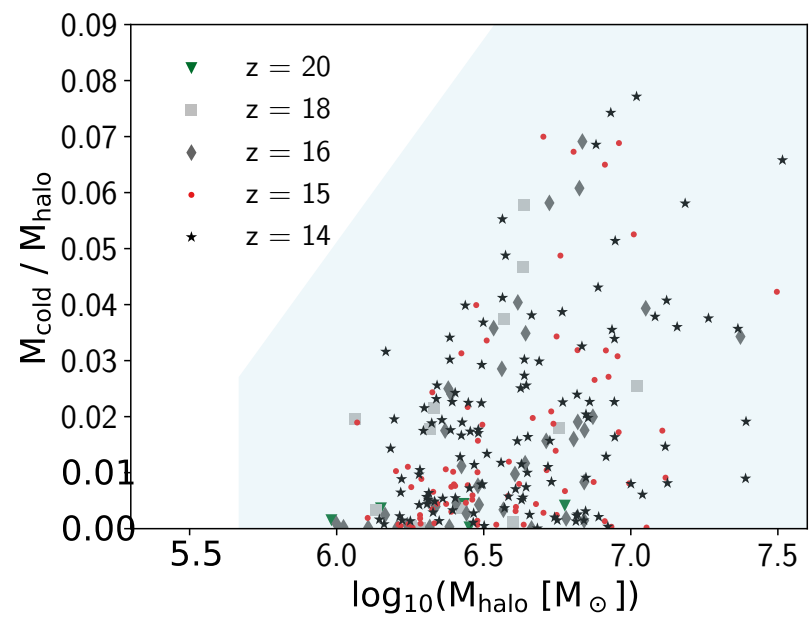

Figure 10. Cold gas fraction $M_{\text {cold }} / M_{\text {halo }}$ as a function of halo mass for the simulation with a streaming velocity of $1 \sigma$ for redshifts in the range $14 \leqslant z \leqslant 20$. Values are only plotted for haloes that contain at least one cold gas cell. The shaded region shows the parameter space occupied by simulation v0.

mass function is larger than that in the run of Naoz, Yoshida \& Gnedin (2012) with a normal $\sigma_{8}=0.82$. This may be due to the different way in which we define halo masses compared to their study: they consider only gas and dark matter within the virial radius, while we consider the mass of the whole structure found by the friends-of-friend algorithm.

Our study goes beyond these existing studies as we are able to follow the collapse of gas to cold, dense structures in the centre of a halo. In Figures 10, 11 and 12 we show the cold mass fraction $M_{\text {cold }} / M_{\text {halo }}$ against halo mass $M_{\text {halo }}$ for the three different streaming velocity simulations. Compared to the case of zero streaming in Figure 5 , one can immediately see that the number of haloes in the figures decreases with increasing streaming velocity. This effect cannot be reduced to the decrease in halo mass function alone. In regions with high streaming velocity, cold gas can only assemble in 


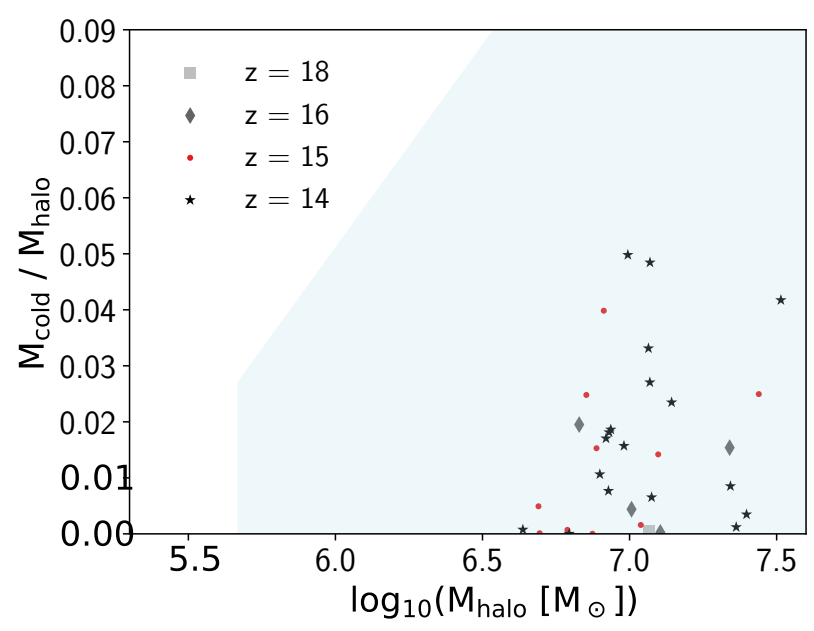

Figure 11. Cold gas fraction $M_{\text {cold }} / M_{\text {halo }}$ as a function of halo mass for the simulation with a streaming velocity of $2 \sigma$ for redshifts in the range $14 \leqslant z \leqslant 18$. Values are only plotted for haloes that contain at least one cold gas cell. The shaded region shows the parameter space occupied by simulation v0.

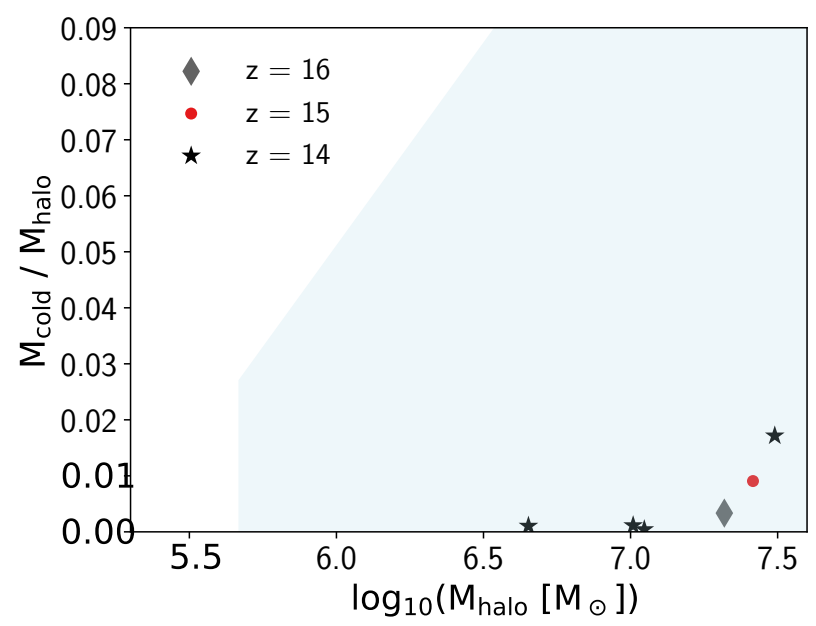

Figure 12. Cold gas fraction $M_{\text {cold }} / M_{\text {halo }}$ as a function of halo mass for the simulation with a streaming velocity of $3 \sigma$ in the small box for redshifts in the range $14 \leqslant z \leqslant 16$. Values are only plotted for haloes that contain at least one cold gas cell. The shaded region shows the parameter space occupied by simulation v0. (Note: as there are only six data points in this figure, we increase the size of the markers compared to Figures 510 and 11).

more massive objects than in regions with zero streaming. This can be seen from the same figures, as the minimum halo mass for cold gas shifts to higher values.

As in the zero streaming case, we can quantify this by determining $M_{\text {halo,min }}$ and $M_{\text {halo,50\% }}$ for each simulation at each output redshift (Figure 13). We see that as the streaming velocity increases, the appearance of cold gas in the simulation is delayed: in run v0, the first cold, dense gas appears at $z \sim 26$, whereas the corresponding redshifts for runs $\mathrm{v} 1$, $\mathrm{v} 2$, and $\mathrm{v} 3$ are $z=20,18$, and 17 , respectively.

Increasing the streaming velocity also increases both $M_{\text {halo,min }}$ and $M_{\text {halo, } 50 \%}$. The increase in these values is not

\begin{tabular}{lrr} 
Name & $M_{\text {halo,min }}$ & $M_{\text {halo, } 50 \%}$ \\
\hline v0 & 0.48 & 1.63 \\
v1 & 1.21 & 3.93 \\
v2 & 6.08 & 11.27 \\
v3 & 13.78 & 20.02 \\
v3_big & 10.0 & 37.9
\end{tabular}

Table 2. Minimum halo mass and halo mass above which more than $50 \%$ of all haloes contain cold gas in units of $10^{6} \mathrm{M}_{\odot}$.

directly proportional to the increase in the streaming velocity, but in general, each time we increase $v_{\text {stream }}$ by $\sigma_{\text {rms }}$, both masses increase by a factor of a few. In Table 2 , we list the values of these masses, averaged over redshifts, for each simulation.

Simulation v3 contains only one halo with cold, dense gas at redshift $z=17$ and three more at our final redshift $z=14$. The apparent increase of the minimum halo mass between redshifts $z=17$ and 14 is a result of this halo growing in mass and therefore of the small sample size. We therefore include the results from the large box v3_big in Figure 13 Thanks to the larger simulation volume, we form many more and also more massive objects in this simulation. The first halo in this simulation to contain cold gas forms at redshift $z=20$.

For a $1 \sigma_{\text {rms }}$ streaming velocity, Greif et al. (2011) found an increase in the minimum mass for cooling and collapse of around a factor of three. This is in good agreement with our results: we find an increase in the minimum halo mass by a factor of 2.5 and in the average halo mass by a factor of 2.4. They also found that the onset of star formation in the halo that they studied was delayed by around $\Delta z \sim 4$. As we do not track the evolution of individual haloes in our simulations, we cannot easily compare this value with the length of time that cooling and star formation is delayed in any particular halo in our simulation. However, it is interesting to note that in run v0, cold gas is first present at $z=26$, while in run $\mathrm{v} 1$, cold gas is first present at $z=20$, i.e. the appearance of cold gas anywhere in the simulation volume is delayed by $\Delta z \sim 6$. Overall, therefore, there seems to be reasonable agreement between the results of Greif et al. (2011) and our own results. However, Greif et al. (2011) did not investigate the impact of higher- $\sigma$ streaming velocities. In addition, studies like Greif et al. (2011) and Stacy, Bromm \& Loeb (2011) examined only the most massive object forming in their simulation volume, rather than the larger statistical sample examined here.

In Figure 14 we compare our minihaloes to literature data and the model suggested by Fialkov et al. (2012). We plot the circular velocity $v_{\text {circ }}$ of each of our simulated haloes at the time that it first contains cold gas versus the value of the streaming velocity at the same time. We define the circular velocity for our haloes as

$$
v_{\mathrm{circ}}=\sqrt{\frac{G M_{\mathrm{vir}}}{R_{\mathrm{vir}}}}
$$

where $G$ is the gravitational constant $G, M_{\text {vir }}$ is the virial mass and $R_{\text {vir }}$ is the virial radius found by the friends-offriends algorithm. We evaluate the circular velocity at the redshift $z$, when a minihalo fulfills our criterion for cold, dense gas for the first time, mimicking collapse of that halo. 


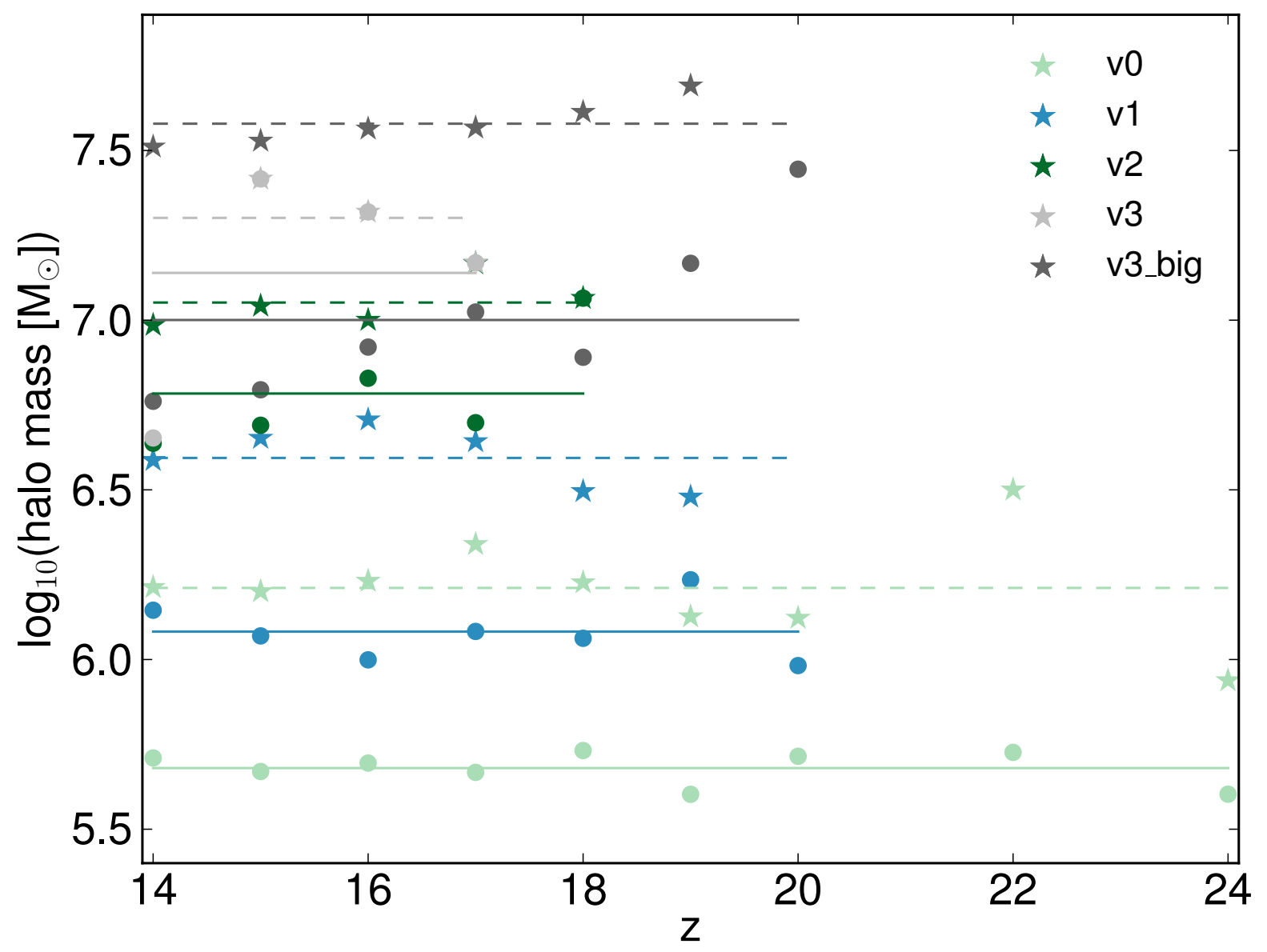

Figure 13. Halo mass of the first halo to contain cold gas (circles) and halo threshold mass above which more than $50 \%$ of all haloes contain cold gas (stars) as a function of redshift. The results for all simulations are shown here. Different colours indicate different streaming velocities. Solid lines show the average mass of the first halo to contain cold gas $\left(M_{\text {halo,min }}\right)$ for the different streaming velocities. Dashed lines show the average halo mass above which $50 \%$ of the haloes contain cold gas $\left(M_{\text {halo, } 50 \%}\right)$ for the same runs.

The streaming velocity at that redshift is given by

$$
v_{\text {stream }}(z)=v_{\text {stream }, 0}\left(\frac{1+z}{1+z_{\text {init }}}\right),
$$

where our streaming velocities have values of $0-18 \mathrm{~km} \mathrm{~s}^{-1}$ at the initial redshift of $z_{\text {init }}=200$.

Fialkov et al. (2012) suggest using an "effective" circular velocity threshold for star formation, based on the minimum circular velocity required for efficient cooling in the absence of streaming, $v_{\text {circ }, 0}$, and the streaming velocity $v_{\text {stream }}(z)$ :

$$
v_{\text {circ }}^{2}=v_{\text {circ }, 0}^{2}+\left[\alpha \times v_{\text {stream }}(z)\right]^{2},
$$

where $\alpha$ is a free parameter to be fit by comparison with simulation results. Fialkov et al. (2012) find an optimal fit to the data from Stacy, Bromm \& Loeb (2011) and Greif et al. (2011) is given by $v_{\text {circ }, 0}=3.714 \mathrm{~km} \mathrm{~s}^{-1}$ and $\alpha=4.015$. We include this line in Figure 14 All of our simulations have circular velocities slightly higher than this "effective" velocity, but it gives a good lower limit for simulations v0, v1, v3 and v3_big. Our data are in the middle of the early simulations by Stacy, Bromm \& Loeb (2011) and Greif et al. (2011) and recent results by Hirano et al. (2018). As the Stacy, Bromm \& Loeb (2011) and Greif et al. (2011) studies select haloes that are the first objects in the simulations that collapse, it is unsurprising that we find that they lie at the bottom end of our distribution of $v_{\text {circ }}$ : objects in their simulations that would have required a larger $v_{\text {circ }}$ to collapse would also have tended to form slightly later and hence would not have been selected. In the case of the Hirano et al. (2018) study, the halo they study is undergoing a merger process. This delays the centre from becoming dense and cold until the halo reaches higher masses. However, our simulation results do provide strong support for their contention that in runs with large streaming velocities, $v_{\text {circ }}$ is a poor predictor of whether or not cooling and collapse can occur in a given minihalo.

\section{CONCLUSIONS}

We have performed five cosmological hydrodynamical simulations using the code AREPO, including a primordial chemistry network. They target different regions of the Universe with zero, 1, 2 and $3 \sigma_{\mathrm{rms}}$ streaming velocity to understand the influence of the streaming velocity value on first star 


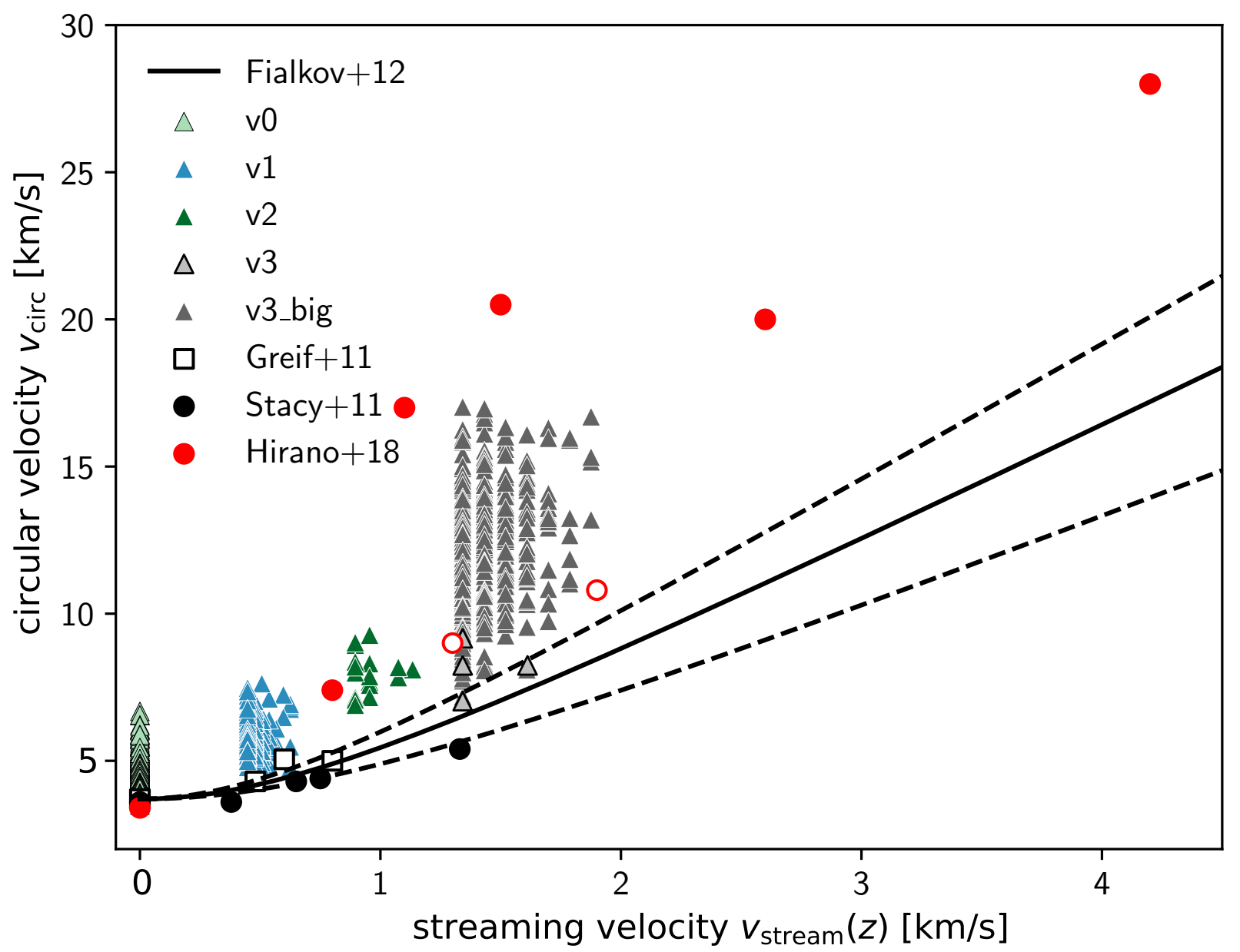

Figure 14. Circular velocity $v_{\text {circ }}$ of all haloes in the simulations as a function of the streaming velocity $v_{\text {stream }}$ at the redshift of collapse. Data from the literature are included for comparison. The red circles show data from Hirano et al. (2018), where the open red circles show two collapsing minihaloes before the collapse was halted and delayed due to a minihalo merger and the filled circles the final collapsed haloes. The black, open squares show data from Greif et al. (2011) and the black, filled circles show data from Stacy, Bromm \& Loeb (2011). The black lines show the corresponding fits from Fialkov et al. (2012), where the solid line includes both data sets and the dashed lines one dataset each.

formation. Our employed resolution is very high throughout the box, leading to thousands of well-resolved minihaloes.

In a region of the Universe with zero streaming velocity, we find that in order for gas to cool within a minihalo, its mass must exceed a minimum mass scale $M_{\text {halo,min }} \approx$ $4.8 \times 10^{5} \mathrm{M}_{\odot}$. However, not all minihaloes above this minimum mass contain cool gas. The fraction of minihaloes containing cool gas increases smoothly with increasing minihalo mass from close to zero at $M \sim M_{\text {halo,min }} \sim 1$ at $M \sim 10^{7} \mathrm{M}_{\odot}$. A majority of minihaloes host cold gas for minihalo masses above $M_{\text {halo, } 50 \%} \approx 1.6 \times 10^{6} \mathrm{M}_{\odot}$. While the minimum halo mass for cold, dense gas depends to some extent on our choice of density threshold, the value of $M_{\text {halo, } 50 \%}$ is much more robust. In contrast to predictions from analytical studies, we do not see a dependence of these characteristic mass scales on redshift. In this, our results agree well with the simulation results of Yoshida et al. (2003), although we find a slightly different minimum mass scale, likely due to the differences in the chemical and cooling model employed in the different simulations.
In regions of the Universe with non-zero streaming velocity, we find a substantial increase in both the minimum halo mass and the average halo mass for the formation of cold, dense gas in a halo. All these masses can be found in Table 2 and are redshift-independent over the range of redshifts studied here. For each $\sigma_{\text {rms }}$ increase in the streaming velocity, we find a factor of a few increase in both $M \sim M_{\text {halo,min }}$ and $M_{\text {halo,50\%. Importantly, this }}$ means that in regions of the Universe with streaming velocities $v_{\text {stream }} \geqslant 3 \sigma_{\mathrm{rms}}$, star formation occurs almost entirely in haloes with $T_{\mathrm{vir}}>10^{4} \mathrm{~K}$, the so-called "atomic cooling haloes" (see also Schauer et al. 2017). The "delay" of star formation in regions of non-zero streaming velocities is purely indirect in our models due to hierarchical structure formation, as the more massive haloes form later.

Fialkov et al. (2012) have proposed that there is a simple relationship between the minimum circular velocity of a halo hosting cold gas and the streaming velocity at the time that the halo formed. Their model uses an "effective" circular velocity (a weighted mean of the circular velocity and 
the streaming velocity at the redshift of halo collapse) as a criterion for Pop III star formation. Our results are broadly consistent with their prediction, if it is interpreted as a necessary but not sufficient condition for cooling: no haloes with circular velocities below their prediction host cold gas, but many haloes with circular velocities above their prediction are also devoid of cool gas. At any particular $v_{\text {stream }}$, there is at least a factor of two scatter in the circular velocity required for a halo to host cool gas. At low $v_{\text {stream }}$, our results are in good agreement with the simulations of Stacy, Bromm \& Loeb (2011), Greif et al. (2011) and Hirano et al. (2018). But at higher $v_{\text {stream, we find cold gas at somewhat }}$ smaller values of $v_{\text {circ }}$ than Hirano et al. (2018). We would like to point out that Hirano et al. (2018) study the first halo forming in a $10 \mathrm{Mpc}$ box. This object is undergoing a merger process that halts the collapse, and the halo first grows in mass before its centre becomes cold and dense. This may explain the difference between Hirano et al. (2018) and our work.

Our results have important implications for the evolution of the cosmological star formation rate at very high redshifts. In the regime where most Pop III stars are forming in minihaloes, increasing the mass threshold for efficient $\mathrm{H}_{2}$ cooling results in a significant reduction in the star formation rate. Therefore, in this regime we would expect the star formation rate at very early times to be inversely correlated with the strength of the streaming velocity. A thorough exploration of the consequences of this is beyond the scope of our current paper, and so we restrict ourselves here to a single example.

The statistical properties of minihaloes are also of interest. In the case of no streaming velocity, the spin distribution of present day galaxies (see e.g. Teklu et al. 2015) follows the same shape as the spin distribution in minihaloes de Souza et al. 2013 Sasaki et al. 2014, Hirano et al. 2014). A rotating disk of cold, dense gas forms in the centre that is however unconnected to the angular momentum on larger scales (Druschke et al. 2018). The spin of the gas increases with streaming velocities (Chiou et al. 2018), and it needs to be investigated how strongly this affects the cold star forming gas in the centre of the halo.

The first measurement of a $21 \mathrm{~cm}$ signal at high redshift (Bowman et al. 2018) by the EDGES experiment reveals a very strong absorption signal, hinting at coupling of gas and dark matter to cool the gas to very low temperatures at redshifts $z \sim 15-20$ (Barkana 2018; Fialkov, Barkana \& Cohen 2018). As the proposed cross-section of baryon-dark matter coupling critically depends on the relative velocity between the gas and the dark matter, streaming velocities play an important role in determining the overall effectiveness of this coupling. Regions with higher streaming velocities will have weaker coupling between baryons and dark matter, and hence slightly higher gas temperatures (Barkana 2018). However, our results demonstrate that cooling of gas in low mass minihaloes will also be strongly suppressed in these regions, resulting in a later onset of star formation and hence plausibly also a later coupling of the $21 \mathrm{~cm}$ spin temperature with the gas temperature. The impact of this effect on the sky-averaged spin temperature at high redshift or on the spatial variation in $T_{\text {spin }}$ in this scenario has yet to be investigated, but will be important for the interpretation not only of the current EDGES result (Bowman et al.
2018), but also the results we expect to obtain with the next generation of telescopes, such as SKA-low or HERA. Detailed knowledge of the influence of streaming velocities on the formation of the first stars is a prerequisite for the correct astrophysical interpretation of the $21 \mathrm{~cm}$ absorption signal and its implications for our understanding of the nature and properties of dark matter.

\section{ACKNOWLEDGMENTS}

The authors acknowledge useful comments from the anonymous referee that helped to improve the quality of the paper. The authors would like to thank Volker Bromm, Mattis Magg, Thomas Greif and Anastasia Fialkov for stimulating discussions. We are grateful to Volker Springel and his group for giving us access to and their help with the code AREPO. Support for this work was provided by NASA through the NASA Hubble Fellowship grant HST-HF251418.001-A awarded by the Space Telescope Science Institute, which is operated by the Association of Universities for Research in Astronomy, Inc., for NASA, under contract NAS5-26555. All authors acknowledge support from the European Research Council under the European Community's Seventh Framework Programme (FP7/2007 - 2013) via the ERC Advanced Grant "STARLIGHT: Formation of the First Stars" (project number 339177). SCOG and RSK also acknowledge support from the Deutsche Forschungsgemeinschaft via SFB 881, "The Milky Way System" (subprojects B1, B2 and B8) and SPP 1573 , "Physics of the Interstellar Medium" (grant number GL 668/2-1). The authors gratefully acknowledge the Gauss Centre for Supercomputing for funding this project by providing computing time on the GCS Supercomputer SuperMUC at the Leibniz Supercomputing Centre under projects pr92za and pr74nu. The authors acknowledge support by the state of BadenWürttemberg through bwHPC and the German Research Foundation (DFG) through grant INST 35/1134-1 FUGG.

\section{REFERENCES}

Ahn K., Iliev I. T., Shapiro P. R., Mellema G., Koda J., Mao Y., 2012, ApJ, 756, L16

Ahn K., Smith B. D., 2018, ArXiv e-prints

Aldrovandi S. M. V., Pequignot D., 1973, A\&A, 25, 137

Badnell N. R., 2006, A\&A, 447, 389

Barkana R., 2018, Nature, 555, 71

Barkana R., Loeb A., 2002, ApJ, 578, 1

Barnes J., Hut P., 1986, Nature, 324, 446

Bowman J. D., Rogers A. E. E., Monsalve R. A., Mozdzen T. J., Mahesh N., 2018, Nature, 555, 67

Bromm V., 2013, Reports on Progress in Physics, 76, 112901

Chiaki G., Susa H., Hirano S., 2018, ArXiv e-prints 1801.01583

Chiou Y. S., Naoz S., Marinacci F., Vogelsberger M., 2018, MNRAS, 481, 3108

Ciardi B., Scannapieco E., Stoehr F., Ferrara A., Iliev I. T., Shapiro P. R., 2006, MNRAS, 366, 689

Clark P. C., Glover S. C. O., Klessen R. S., Bromm V., 2011, ApJ, 727, 110 
Streaming velocities at first star formation

Coppola C. M., Longo S., Capitelli M., Palla F., Galli D., 2011, ApJS, 193, 7

Crosby B. D., O'Shea B. W., Smith B. D., Turk M. J., Hahn O., 2013, ApJ, 773, 108

de Souza R. S., Ciardi B., Maio U., Ferrara A., 2013, MNRAS, 428, 2109

Druschke M., Schauer A. T. P., Glover S. C. O., Klessen R. S., 2018, MNRAS, 481, 3266

Eisenstein D. J., Hu W., 1998, ApJ, 496, 605

Fialkov A., 2014, International Journal of Modern Physics D, 23, 1430017

Fialkov A., Barkana R., 2014, MNRAS, 445, 213

Fialkov A., Barkana R., Cohen A., 2018, ArXiv e-prints; arXiv:1802.10577

Fialkov A., Barkana R., Tseliakhovich D., Hirata C. M., 2012, MNRAS, 424, 1335

Fuller T. M., Couchman H. M. P., 2000, ApJ, 544, 6

Galli D., Palla F., 1998, A\&A, 335, 403

Glover S., 2013, in Astrophysics and Space Science Library, Vol. 396, Astrophysics and Space Science Library, Wiklind T., Mobasher B., Bromm V., eds., p. 103

Greif T. H., White S. D. M., Klessen R. S., Springel V., 2011, ApJ, 736, 147

Hahn O., Abel T., 2011, MNRAS, 415, 2101

Haiman Z., Abel T., Madau P., 2001, ApJ, 551, 599

Hartwig T., Clark P. C., Glover S. C. O., Klessen R. S., Sasaki M., 2015b, ApJ, 799, 114

Hartwig T., Glover S. C. O., Klessen R. S., Latif M. A., Volonteri M., 2015a, MNRAS, 452, 1233

Hirano S., Hosokawa T., Yoshida N., Umeda H., Omukai K., Chiaki G., Yorke H. W., 2014, ApJ, 781, 60

Hirano S., Yoshida N., Sakurai Y., Fujii M. S., 2018, ApJ, 855,17

Hirata C. M., Padmanabhan N., 2006, MNRAS, 372, 1175

Hummel J. A., Pawlik A. H., Milosavljević M., Bromm V., 2012, ApJ, 755, 72

Latif M. A., Niemeyer J. C., Schleicher D. R. G., 2014, MNRAS, 440, 2969

Mac Low M.-M., Ferrara A., 1999, ApJ, 513, 142

Machacek M. E., Bryan G. L., Abel T., 2001, ApJ, 548, 509

Magg M., Hartwig T., Agarwal B., Frebel A., Glover S. C. O., Griffen B. F., Klessen R. S., 2018, MNRAS, 473, 5308

Magg M., Hartwig T., Glover S. C. O., Klessen R. S., Whalen D. J., 2016, MNRAS, 462, 3591

Naoz S., Barkana R., 2005, MNRAS, 362, 1047

Naoz S., Barkana R., Mesinger A., 2009, MNRAS, 399, 369

Naoz S., Yoshida N., Barkana R., 2011, MNRAS, 416, 232

Naoz S., Yoshida N., Gnedin N. Y., 2012, ApJ, 747, 128

Naoz S., Yoshida N., Gnedin N. Y., 2013, ApJ, 763, 27

O’Leary R. M., McQuinn M., 2012, ApJ, 760, 4

Planck Collaboration et al., 2016, A\&A, 594, A13

Popa C., Naoz S., Marinacci F., Vogelsberger M., 2016, MNRAS, 460, 1625

Sasaki M., Clark P. C., Springel V., Klessen R. S., Glover S. C. O., 2014, MNRAS, 442, 1942

Schauer A. T. P., Regan J., Glover S. C. O., Klessen R. S., 2017, MNRAS, 471, 4878

Seljak U., Zaldarriaga M., 1996, ApJ, 469, 437

Shapiro P. R., Ahn K., Alvarez M. A., Iliev I. T., Martel H., Ryu D., 2006, ApJ, 646, 681
Smith B. D., Wise J. H., O'Shea B. W., Norman M. L., Khochfar S., 2015, MNRAS, 452, 2822

Springel V., 2010, MNRAS, 401, 791

Springel V., White S. D. M., Tormen G., Kauffmann G., 2001, MNRAS, 328, 726

Stacy A., Bromm V., Loeb A., 2011, ApJ, 730, L1

Tanaka T. L., Li M., 2014, MNRAS, 439, 1092

Tegmark M., Silk J., Rees M. J., Blanchard A., Abel T., Palla F., 1997, ApJ, 474, 1

Teklu A. F., Remus R.-S., Dolag K., Beck A. M., Burkert A., Schmidt A. S., Schulze F., Steinborn L. K., 2015, ApJ, 812, 29

Trenti M., Stiavelli M., 2009, ApJ, 694, 879

Tseliakhovich D., Barkana R., Hirata C. M., 2011, MNRAS, 418, 906

Tseliakhovich D., Hirata C., 2010, Phys. Rev. D, 82, 083520

Wise J. H., Turk M. J., Norman M. L., Abel T., 2012, ApJ, 745,50

Yoshida N., Abel T., Hernquist L., Sugiyama N., 2003, ApJ, 592,645

Yue B., Ciardi B., Scannapieco E., Chen X., 2009, MNRAS, 398,2122

\section{APPENDIX A: UPDATES TO THE CHEMICAL MODEL}

The primordial chemistry model used in this study is based on the model implemented in AREPO by Hartwig et al. (2015a), but has been updated in several respects. The most significant difference is the inclusion of a simplified treatment of deuterium chemistry designed to track the formation and destruction of HD. Our treatment of the deuterium chemistry follows that in Clark et al. (2011) and full details can be found in that paper. In the interests of brevity, we do not repeat them here.

The second update involves our treatment of the dielectronic recombination of ionized helium, $\mathrm{He}^{+}$. In Hartwig et al. (2015a) we used a rate for this process taken from Aldrovandi \& Pequignot (1973). In our present study, we use instead the more recent determination by Badnell (2006). In practice, we expect this change to have no significant impact on our results, as at the gas temperatures probed in this study, very little ionized helium is present, and the small amount that is created recombines primarily via radiative recombination rather than dielectronic recombination.

Finally, we have also updated our treatment of $\mathrm{H}^{-}$photodetachment. In all of our runs, we account for photodetachment of $\mathrm{H}^{-}$by $\mathrm{CMB}$ photons. The rate for this process is given by the sum of two contributions, one describing the influence of the thermal CMB photons and a second describing the influence of the non-thermal radiation background produced by cosmic recombination at $z \sim 1100$ ( $\mathrm{Hi}-$ rata \& Padmanabhan 2006). For the thermal contribution, we adopt the following rate coefficient from Galli \& Palla (1998),

$$
R_{\mathrm{H}^{-}, \mathrm{th}}=0.11 T_{\mathrm{r}}^{2.13} \exp \left(\frac{-8823 \mathrm{~K}}{T_{\mathrm{r}}}\right) \mathrm{s}^{-1},
$$

where $T_{\mathrm{r}}=T_{\mathrm{CMB}}=2.73(1+z) \mathrm{K}$. For the non-thermal 


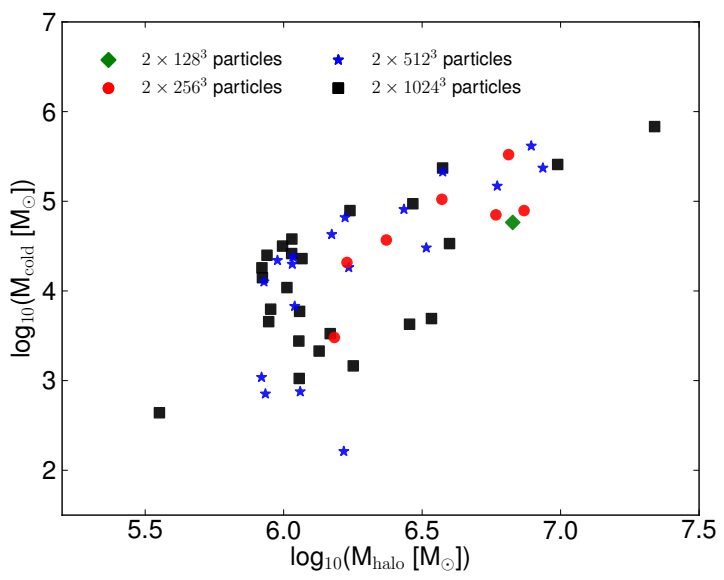

Figure B1. Resolution test: different number of particles and Voronoi cells for a cosmological box with the side length of $500 \mathrm{ckpc} / h$. There are many fewer red circles $\left(2 \times 256^{3}\right.$ particles $)$ and only one green diamond $\left(2 \times 128^{3}\right.$ particles $)$ than blue stars $\left(2 \times 512^{3}\right.$ particles $)$ or black squares $\left(2 \times 1024^{3}\right.$ particles $)$. Except for one halo at $\approx 10^{5.6} \mathrm{M}_{\odot}$, the onset of efficient cooling at a halo mass $\approx 10^{5.8} \mathrm{M}_{\odot}$ is the same for the $2 \times 512^{3}$ and $2 \times 1024^{3}$ runs.

contribution, we adopt the expression (Coppola et al. 2011)

$$
R_{\mathrm{H}^{-}, \mathrm{nth}}=8 \times 10^{-8} T_{\mathrm{r}}^{1.3} \exp \left(\frac{-2300 \mathrm{~K}}{T_{\mathrm{r}}}\right) \mathrm{s}^{-1} .
$$

The total $\mathrm{H}^{-}$photodetachment rate is simply the sum of these two terms.

\section{APPENDIX B: NUMERICAL CONVERGENCE}

We want to provide robust results that are numerically converged. Therefore, we test our fiducial resolution of $2 \times 1024^{3}$ particles in a $(1 \mathrm{cMpc} / h)^{3}$ volume against larger and smaller mass resolutions with otherwise identical initial conditions. For simulations with box length of $500 \mathrm{ckpc} / h$, we vary the number of particles from $2 \times 128^{3}$ to $2 \times 1024^{3}$ in steps of eight, so that the mass resolution changes between each simulation by a factor of 8 . For our convergence study, we consider the case with zero streaming velocities, as this run has the lowest halo mass threshold for efficient $\mathrm{H}_{2}$ cooling. If the lowest mass haloes containing cold gas are well resolved in this simulation, the more massive haloes containing cold gas in the simulations with non-zero streaming velocities should certainly be adequately resolved.

In Figure B1 we show the cold gas mass against halo mass for the four realizations. One can see that for the two lower resolutions of $2 \times 128^{3}$ particles and $2 \times 256^{3}$ particles, the cold gas mass is lower than for the two higher resolution cases. Our fiducial resolution of $2 \times 512^{3}$ particles (which is equivalent to $2 \times 1024^{3}$ particles in our volume of $1 \mathrm{cMpc}^{3}$ in the production runs) agrees well with a even higher resolution of $2 \times 1024^{3}$ particles. We are therefore confident that our simulations are well converged.

These results also help to motivate our choice of simulation volume in our large box simulation, v3_big. They demonstrate that in order to properly resolve cooling in these haloes, we to resolve each halo with at least a few

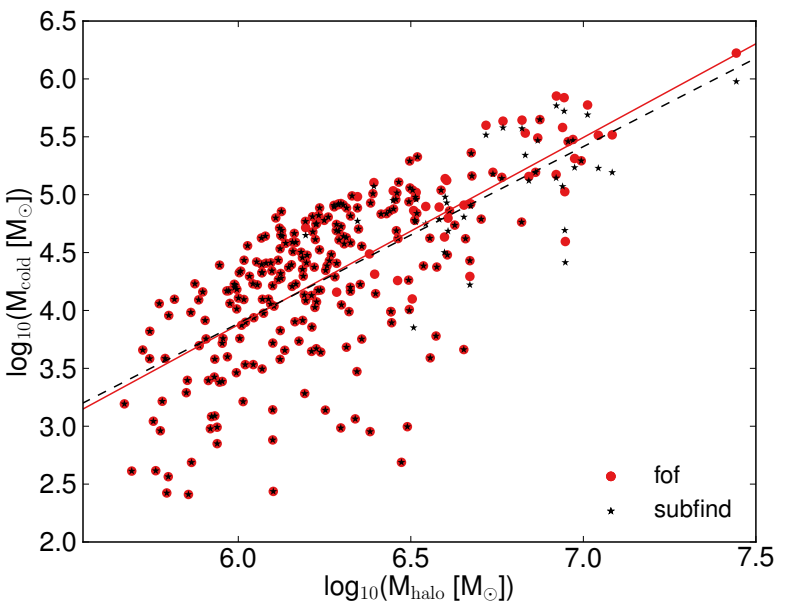

Figure C1. Cold gas mass against halo mass for the simulation without streaming at redshift $z=15$. We show the cold gas mass selected by two different halo finders: cold gas found in the whole friends-of-friends (fof) structure (red circles) and cold gas only found in the most massive subhalo (black stars). In most cases, there is very good agreement, but in the most massive haloes, the fof halo finder tends to recover slightly larger cold gas masses than subfind.

thousand dark matter particles. In our large box simulation, low mass haloes with $M_{\text {halo }} \sim 10^{6} \mathrm{M}_{\odot}$ are resolved with only around a hundred particles, and so cooling is not modelled accurately in these haloes. However, we know from run v3 that the minimum mass required for cooling in a simulation with a $3 \sigma$ streaming velocity is increased by over an order of magnitude, to $M \sim 10^{7} \mathrm{M}_{\odot}$, and haloes of this mass are resolved by at least a thousand particles. Therefore, the lowest mass haloes in which efficient $\mathrm{H}_{2}$ cooling occurs in run v3_big are resolved by a comparable number of dark matter particles to the lowest mass haloes in which efficient $\mathrm{H}_{2}$ cooling occurs in run v0.

\section{APPENDIX C: COMPARISON OF FRIENDS-OF-FRIENDS RESULTS WITH SUBFIND}

In the high-redshift Universe, minihaloes tend not to be spherical but instead are very elongated and irregular (see e.g. Sasaki et al. 2014). Our results could therefore depend on the halo finding algorithm. In this Appendix, we provide a check to show that our results are independent of the halo selection algorithm. In this paper, we have considered all cold gas particles that were associated to a halo by the standard friends-of-friends (fof) algorithm. Another possibility is to consider only gas that belongs to the most bound subhalo in the fof structure. For this test, we use the halo finding algorithm subfind (Springel et al. 2001).

In Figure C1. we show the cold gas mass as a function of halo mass for the simulation without streaming at redshift $z=15$ (red circles). Overplotted are the cold gas masses in the most bound subhaloes (black stars). The cold gas mass found in the most bound subhalo agrees very well with the cold gas mass found in the whole fof structure. At the highest halo masses, some haloes contain more cold gas when 


\begin{tabular}{lcccc} 
Name & $a$ & $b$ & $a$ & $b$ \\
$z$ & 15 & 15 & 20 & 20 \\
\hline v0 & 1.6 & 5.8 & 1.3 & 4.0 \\
v1 & 1.8 & 7.2 & 1.2 & 3.8 \\
v2 & 3.1 & 17.0 & - & - \\
v3_big & 1.7 & 7.7 & 2.8 & 16.0 \\
\hline
\end{tabular}

Table D1. Cold gas mass - halo mass relation for all simulations at redshifts 15 and 20. Simulation v2 does not have enough data points at $z=20$ to provide a meaningful fit. The same is true for simulation v3 at both redshifts, and so we omit it from the table.

considering the whole fof structure instead of only the most bound subhalo, but this difference does not affect any of the main results of the paper. We therefore conclude that for this study, our results are not sensitive to our choice of halo finding algorithm.

\section{APPENDIX D: COLD MASS - HALO MASS RELATION}

In Section 3.1 we have provided a fit for the cold gas mass - halo mass relation. As can be seen in Figure2 the scatter is significant and should not be neglected when using our fitting approximation. Despite this caveat, we provide fits to all our simulations in Table D1 that could be used for semi-analytical calculations. This relation is valid only for the mass range of haloes formed in these simulations, from about $5 \times 10^{5} \mathrm{M}_{\odot}$ to $3 \times 10^{7} \mathrm{M}_{\odot}$ in most cases. The data in this table is given in the form

$$
\log _{10}\left(M_{\text {cold }}\right)=a \times \log _{10}\left(M_{\text {halo }}\right)-b \text {. }
$$

